\title{
A Comparative Study of Impairments Aware Optical Networks Planning Tools
}

\author{
Siamak Azodolmolky, \\ Marianna Angelou, \\ and Ioannis Tomkos \\ Athens Information Technology \\ Athens, Greece \\ Email: \{sazo,mang,itom\}@ait.edu.gr
}

\author{
Annalisa Morea \\ and Yvan Pointurier \\ Alcatel-Lucent, Bell Labs, France \\ Email: annalisa.morea@alcatel-lucent.com, \\ yvan@ieee.org
}

\author{
Josep Solé-Pareta \\ Universitat Politècnica de Catalunya \\ Catalunya, Barcelona, Spain \\ Email: pareta@ac.upc.edu
}

\begin{abstract}
Transparent and translucent optical networks are widely considered as the prime candidates for the core network technology of the future. These networks provide ultra high speed end-to-end connectivity with high quality of service $(\mathrm{QoS})$ and resilience to failures. This will be achieved through appropriate network planning techniques. A downside of transparency, however, is the accumulation of physical layer impairments over long distances, which are difficult to mitigate using purely physical-layer techniques. Considering the impact of physical layer impairments on network planning and operation has received considerable attention from research community. A novel physical layer impairment aware network planning tool is presented in this paper. Its performance is quantitatively compared with results obtained by a state-of-the-art tool under a common network scenario. The differences between the two planning approaches are illustrated and discussed.
\end{abstract}

\section{INTRODUCTION}

The evolution trend of optical networks is a transformation towards higher capacity and lower cost core optical networks [1]. Operators also expect the optical networks to become more agile in order to meet their requirements in terms of fast and automatic reconfiguration. Transparent optical networking is one of the main trends toward network agility, as it incorporates routing and wavelength mechanisms, which are agnostic to the modulation format and/or transmission rates. Transparent networks can also contribute to lower power consumption and heat dissemination by avoiding the unconditional use of regenerators at each network node. However, a downside of transparency, is the accumulation of physical layer impairments over long distances, which are difficult to mitigate using physical-layer related techniques. In optical networks, bandwidth is allocated in the form of lightpaths (i.e., a route and a wavelength). Physical layer impairments accumulate as light propagates through a lightpath.

Due to large coverage of core optical networks, some lightpaths may not be feasible due to an unacceptable final bit error rate (BER) at the destination node. This issue is critical in the planning phase of the network and for the control plane functionality during network operation. Therefore, in order to increase the speed of lightpath establishment, it can be useful to avoid repeated, unsuccessful attempts by enhancing the control plane with an RWA process, which is aware of the impact of physical layer impairments and considers the quality of transmission (QoT) requirements.

To materialize the vision of transparent optical networks, while offering efficient resource utilization and strict quality of service guarantees based on certain service level agreements, the core network should efficiently provide high capacity, fast and flexible provisioning of lightpaths, high reliability, and integrated control plane functionalities.

Considering the physical layer impairments in the network planning phase gives rise to a set of offline Impairments Aware Routing and Wavelength Assignment (IA-RWA) [2] and regenerator placement algorithms. During the planning phase, the traffic demand is already known, enabling the network designer to perform the resource allocation task upfront. To the best of our knowledge this is the first comparative study, in which two different network planning tools (DICONET [3] impairment aware network planning tool (IANPT) and DIAMOND [4]) with different approaches regarding the consideration of the physical impairments are quantitatively compared using a common network scenario and physical constraints framework.

In this work we compare the behavior of two design tools. The DICONET IANPT tool utilizes an accurate but computationally expensive QoT estimator to return network designs with fewer regenerators and physically longer lightpaths than DIAMOND.

This paper is organized as follows. In Section II the description of two network planning tools (i.e., DICONET IANPT and DIAMOND) are presented. The comparative simulation study and its setup is described in Section III. The obtained results and discussions are presented in Section IV and Section $\mathrm{V}$ draws the conclusions of this work.

\section{Impairments Aware Network Planning Tools}

The main functionality of a network planning tool is to receive a traffic demand set along with the network description (topology and/or physical layer) as inputs and to compute a list of lightpaths and possibly some regenerator locations to serve the demand set. An IANPT considers the impact of physical layer impairments while solving the RWA problem. The key building blocks of the DIAMOND and DICONET IANPT 


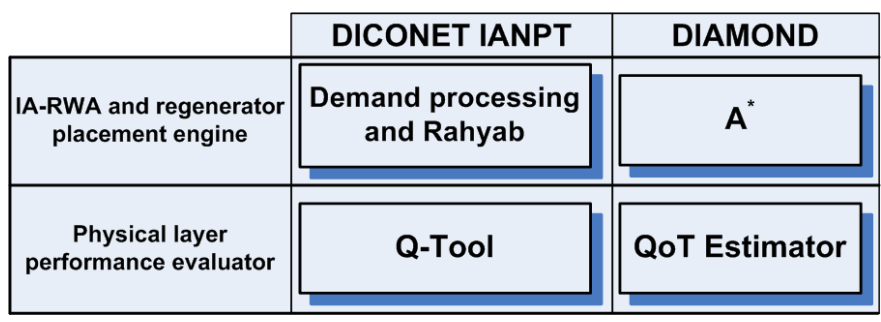

Fig. 1. Structure of DICONET IANPT and DIAMOND.

tools, which are considered in our comparative studies, are depicted in Fig. 1.

The Q-Tool and Rahyab [5] are two key components of the DICONET IANPT. The Q-Tool is a QoT estimator that combines most of the dominant physical impairments of a WDM system into a single figure of merit (the Q-factor). Given all the necessary topological, physical layer characteristics, and current network state, the Q-Tool estimates the Q-factor of a set of lightpaths. The Q-Tool estimates the distortion-induced eye closure that defines the impact of the combined effect of Self Phase Modulation (SPM), Chromatic Dispersion (CD) Filter Concatenation (FC) and Polarization Mode Dispersion (PMD). It also considers the impairments that introduce degradations at the amplitude levels, i.e. Amplified Spontaneous Emission noise (ASE), Cross Phase Modulation (XPM), and Four Wave Mixing (FWM).

The Rahyab module of IANPT receives a demand set, in the form of triplets source, destination, and number of lightpaths and computes corresponding lightpaths for it. The IANPT initially processes the demand set. In case that some of the demands cannot be served transparently, the regenerators will be deployed and the corresponding demands will be transformed into a set of transparent demands. In this step the regenerators will be put in some of the network nodes to make sure that the end-to-end QoT of the signal is acceptable after intermediate regeneration. Thus, the transformed demand set only includes the demands that can be served in transparent mode. After this initial step the IA-RWA engine of IANPT serves the transformed demand set sequentially. The Rahyab module performs a demand pre-processing step and then serves them sequentially. It generates a pool of candidate lightpaths for each demand and from this pool selects the one, which introduces the minimum impact (in terms of QoT) on the currently established lightpaths [5].

DIAMOND is a planning tool that searches for a lightpath between two nodes in a network by means of a layered network graph [4]. The path search is iterative and places a regenerator or wavelength converter whenever it is required (due to QoT or wavelength continuity constraints, respectively). The chosen path is the one having the lesser cost, which is obtained considering the link lengths and the eventual cost associated to intermediary regenerators. The path search is a heuristic based on the $\mathrm{A}^{*}$ algorithm [4]. The traffic demands are considered in the arrival order and they are routed sequentially. A demand is blocked whenever there is no available resource, in terms of either available wavelengths in the fiber or regenerators in an intermediate node. The Q-factor is estimated by a polynomial function (QoT-estimator) considering the accumulation of the main effects degrading the signal propagation, such as ASE, $\mathrm{CD}$ and PMD, FC and nonlinearities. This QoT estimator considers the worst case impact of non-linear impairments due to active neighboring channels. More information about such Q-estimator is in [4]. To consider estimation uncertainties associated to an estimate, a fixed margin is added to the estimated Q-factor [6]. DIAMOND utilizes a path search heuristic based on the $\mathrm{A}^{*}$ algorithm, considering the resource availability and minimum usage of regenerators [4]. During the path search the QoT validation is performed and if the shortest path is not able to have a QoT higher than a certain threshold, while a longer path does, the longer path will be chosen. The same technique is also utilized for the wavelength assignment. In order to guarantee the feasibility of a candidate lightpath, the QoT-estimator considers the worst case scenario for all connections, i.e. all neighbor channels are present.

DICONET IANPT finds the optimum lightpath from the set of candidate lightpaths, considering the degradation due to neighboring lightpaths. The optimum lightpath is the one that introduces the minimum QoT degradation on already established lightpaths. Rahyab intensively uses the Q-Tool to evaluate the QoT of the already established lightpaths in order to admit or reject a new demand. This means that Q-Tool is less pessimistic than the DIAMOND QoT estimator and can enable saving of regenerators. The main advantage of DIAMOND is its computation speed, while DICONET IANPT requires more time to compute the optimum solution.

\section{Simulation Setup}

We selected Deutsche Telekom's national network (DTNet) for our simulation studies. This network has 14 nodes and 23 bidirectional links, with an average node degree of 3.29 and average link length of $186 \mathrm{~km}$. The physical characteristics of DTNet is shown in Fig. 2. We define the offered load in the network as the ratio between the number of lightpath demands divided by the number of pairs of nodes in the network. The unit traffic load corresponds to the demand set where there is on average a lightpath request between each pair of (distinct) source-destination. We studied three traffic load values (i.e. 0.3, 0.6 and 0.8 ), corresponding here to the establishment of 56, 110 and 146 lightpaths. In Fig. 3 the Q factor value (computed by Q-Tool) of 10 shortest paths between all possible pairs of the nodes is depicted. Without considering the impact of other established lightpaths, there is no lightpath with a length longer than $1500 \mathrm{~km}$ and acceptable QoT. There are short lightpaths with Q value lower than threshold (Region 1) and long lightpaths with acceptable Q values (Region 2). This demonstrates the benefit of IA-RWA engines, which are able to find long but feasible lightpaths.

\section{RESUlTS}

IANPT and DIAMOND served all demands without any blocking for all loads. IANPT served all demands without any 


\begin{tabular}{|l|l|}
\hline \multicolumn{1}{|c|}{ Parameter } & \multicolumn{1}{c|}{ Value } \\
\hline Input power & -4 (SSMF), 3 (DCF) $\mathrm{dBm}$ \\
\hline Pre-dispersion compensation & $-85 \mathrm{ps} / \mathrm{nm}$ \\
\hline Span length & $70 \mathrm{~km}$ \\
\hline Dispersion parameter & $17(\mathrm{SSMF}), 80$ (DCF) $\mathrm{ps} / \mathrm{nm} / \mathrm{km}$ \\
\hline Attenuation & $0.23(\mathrm{SSMF}), 0.4$ (DCF) dB/km \\
\hline PMD & $0.1 \mathrm{ps} /(\mathrm{km})^{1 / 2}$ \\
\hline Channel spacing & $50 \mathrm{GHz}$ \\
\hline Amplifier noise figure & $6 \mathrm{~dB}$ \\
\hline Mean under compensated dispersion & $80 \mathrm{ps} / \mathrm{nm}$ per span \\
\hline Q-factor threshold & $15.5 \mathrm{~dB}\left(\mathrm{BER}=10^{-9}\right.$ without FEC) \\
\hline Line rate & $10 \mathrm{Gbps}$ \\
\hline Number of channels per fiber & 16 \\
\hline
\end{tabular}

Fig. 2. Physical characteristics of DTNet.

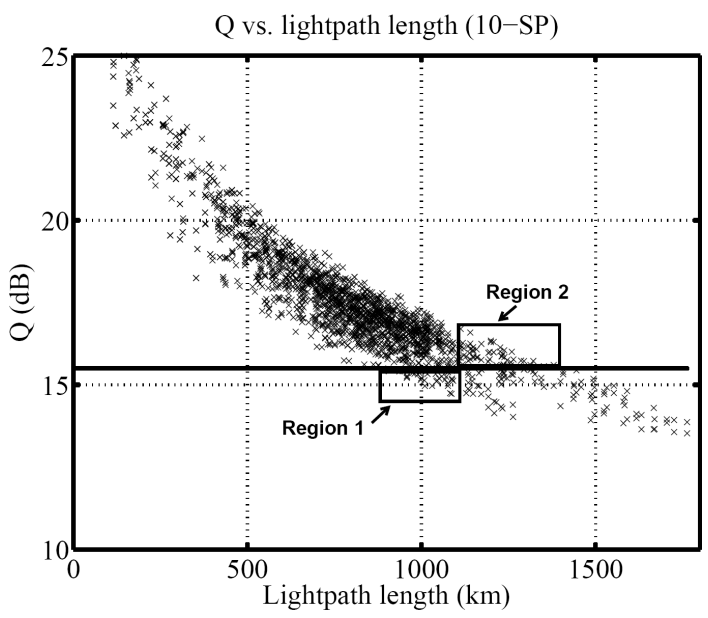

Fig. 3. Benefit of IA-RWA and different solution spaces.

regenerator and there was no need to transform the demand set as a result of initial step of IANPT as described in Section II. However DIAMOND computed a need for 2 and 6 regenerators for load values 0.6 and 0.8 respectively. This is mainly due to the QoT estimator, which is utilized in DIAMOND. The QoT estimator considers the worst case scenario to consider the impact of neighboring channels and therefore the QoT estimation is more pessimistic than DICONET Q-Tool. Indeed DIAMOND utilizes a path search heuristic to find a lightpath with minimum number of regenerators and acceptable QoT value. However, wavelength blocking can occur, which is alleviated by wavelength conversion using regenerators. The Rahyab module of IANPT intensively invokes the Q-Tool to evaluate the performance of each candidate lightpath in order to guarantee the minimum QoT impact of the new lightpath on the currently established ones. Therefore the computation time of IANPT is very high compared to DIAMOND. The computation time of IANPT for load 0.3 was 9 hours while DIAMOND computes the results in $563 \mathrm{~ms}$. The cumulative distribution function of the lightpath length for different loads is depicted in Fig. 4. The distribution of the lightpaths length, is presented in Fig. 5 for all 3 demand sets combined. We can observe from these two figures that the diverse routing

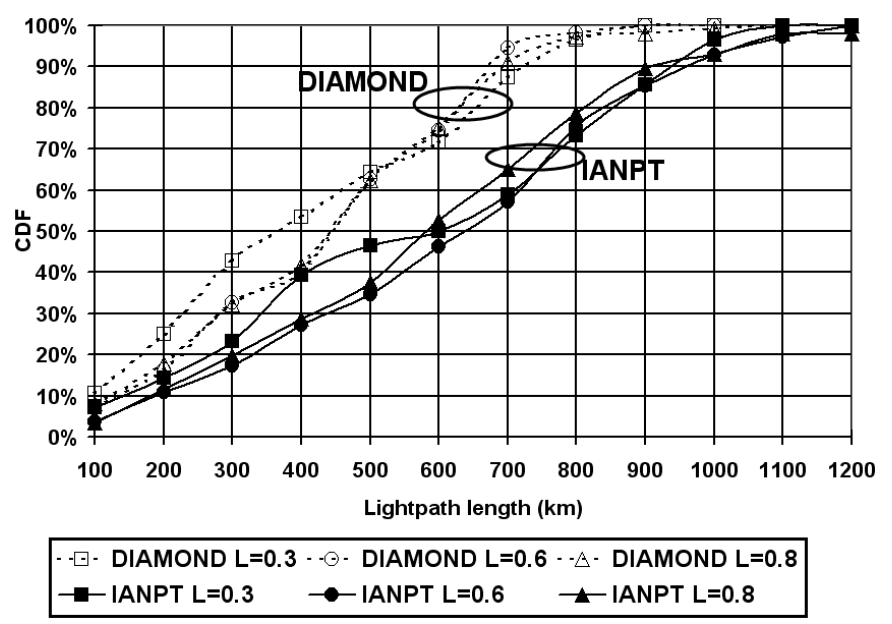

Fig. 4. CDF of lightpath length for different loads.

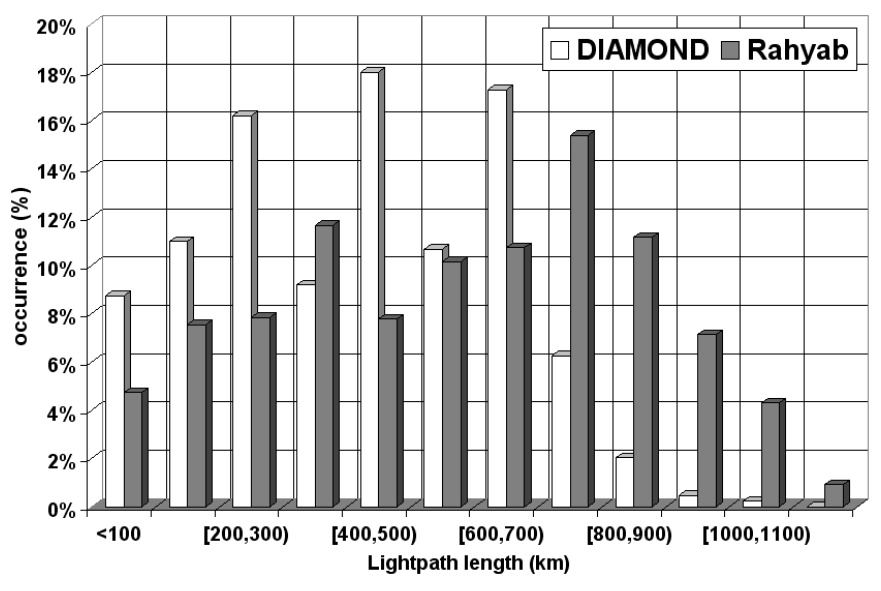

Fig. 5. Distribution of lighpaths length.

engine of IANPT could find longer feasible lightpaths compared to DIAMOND. The average length of the lightpaths in DIAMOND is $419 \mathrm{~km}$ and $572 \mathrm{~km}$ for IANPT. Almost all computed lightpaths by DIAMOND have a length lower that $900 \mathrm{~km}$. IANPT only considers the active lightpaths in order to admit or reject a demand, while DIAMOND (and in particular the QoT estimator) considers a worst case scenario, in which all neighboring lightpaths are active. As argued in Fig. 3 IANPT can find longer feasible lightpaths compared to DIAMOND. Fig. 6 presents the distribution of wavelength usage by DIAMOND and IANPT for a particular demand set (i.e. $\mathrm{Load}=0.3$ ). Rahyab utilizes an adaptive wavelength assignment approach, in which the wavelength of the candidate lightpath is selected in a way that it introduces the minimum impact on the currently established lightpaths. DIAMOND finds a lightpath with fewer regenerators. The $\mathrm{A}^{*}$ routing engine of DIAMOND and the wavelength assignment mechanism rely on the QoT estimator to guarantee the acceptable QoT of the selected lightpath. The Rahyab wavelength usage pattern is adaptive along the available channels per links depending on the network state and some channels are not assigned to any 


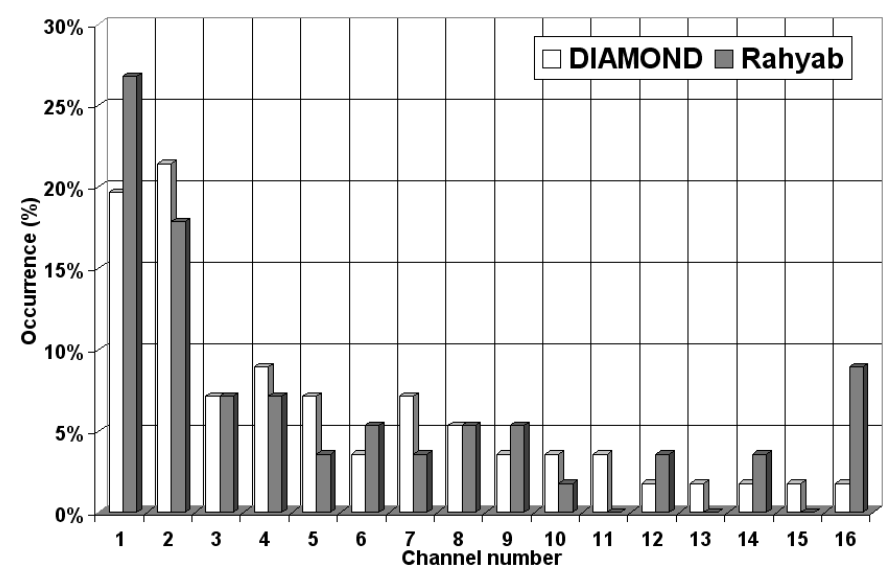

Fig. 6. Frequency of channel usage (Load=0.3).

lightpath. We also observed that for the given demand sets on average the first 10 channels on the links were sufficient for both planning tools to serve $80 \%$ of the demands.

IANPT and DIAMOND rely on different QoT estimators as depicted in Fig. 1. In order to evaluate the quality of the solutions of these tools, we fed the solution of each tool for each demand set to the IANPT's Q-Tool. The average Q value of DIAMOND's solutions is $4 \%$ better than IANPT. The average Q-factor of DIAMOND's solution (over three demand sets) is $28 \mathrm{~dB}$. This is mainly due to the fact that DIAMOND routing module selects shortest paths in general to admit or reject a lightpath.

\section{CONClusion}

The evolution trend of optical networks introduces a need for intelligent and impairments aware network planning tools. DICONET IANPT and DIAMOND are two tools that address this issue. The IANPT adopt an approach, which results in lower number of regenerators and longer feasible lightpaths compared with DIAMOND. However the computation time and complexity of DIAMOND is much lower that IANPT, at the expense of a higher number of required regenerators.

\section{ACKNOWLEDGMENT}

The authors would like to thank the EU ICT FP7-DICONET project for funding this work.

\section{REFERENCES}

[1] J. Berthold, A. A. M. Saleh, L. Blair, and J. M. Simmons, "Optical networking: Past, present, and future," J. Lightw. Technol., vol. 26, no. 9 , pp. 1104-1118, May 2008.

[2] S. Azodolmolky, M. Klinkowski, E. Marin, D. Careglio, J. Solé-Pareta, and I. Tomkos, "A survey on physical layer impairments aware routing and wavelength assignment algorithms in optical networks," Elsevier Computer Networks, vol. 53, no. 7, pp. 926-944, May 2009.

[3] S. Azodolmolky et al., "A dynamic impairment aware networking solution for transparent mesh optical networks," IEEE Commun. Mag., vol. 47, no. 5, pp. 38-47, May 2009.

[4] A. Morea, N. Brogard, F. Leplingard, J. Antona, T. Zami, B. Lavigne, and D. Bayart, "QoT function and A* routing: an optimized combination for connection search in translucent networks," Journal of Optical Networking, vol. 7, no. 1, pp. 42-61, 2008.
[5] S. Azodolmolky, Y. Pointurier, M. Angelou, J. Solé-Pareta, and I. Tomkos, "An offline impairment aware RWA algorithm with dedicated path protection consideration," in Proc. IEEE/OSA OFC/NFOEC, OWII, Mar. 2009.

[6] F. Leplingard, A. Morea, T. Zami, and N. Brogard, "Interest of an adaptive margin for the quality of transmission estimation for lightpath establishment," in Proc. IEEE/OSA OFC/NFOEC, OWI6, Mar. 2006. 